\title{
TESTS DE CAUSALIDAD, DINERO Y RENTA EN ESPAÑA, 1904-1974
}

JOSE LUIS GARCIA RUIZ Universidad Complutense

\section{RESUMEN}

El presente trabajo utiliza el método de los tests de causalidad y lo aplica a la relación entre las fuerzas monetarias y las fuerzas reales de la economia, en el caso espanol, entre 1904 y 1974, utilizando las principales series de dinero y renta disponibles. Los resultados no eaconden su ambigüedad, y ello, aunque se correaponde con las experiencias de otros paises, queda explicado, en buena medida, por el diferente comportamiento en el corto plazo de las series de renta utilizadas.

\section{ABSTRACT}

This eseny applies the causality test methodology to the series of income and money in Spain between 1904 and 1974. The results are not unambiguous, like in other experiences, but in the Spanish case ambiguity could be explained by the short term behavior of income.

\section{INTRODUCCION: MACROECONOMIA Y REALIDAD *}

En 1980 Christopher Sims publicó un extenso artículo en el que enunciaba que uuna vena de profundo escepticismo acerca del valor de los modelos se extiende entre los economistas no comprometidos activamente en su construcción o uson ${ }^{1}$.

Sims resumía el problema como una cuestión de incredulidad en la identificación de los modelos. Aun reconociendo que las restricciones a priori, los problemas de dinámica y la introducción de las expectativas han complica-

* Quisiera agradecer a los profesores Juan Hemández Andreu, Pablo Martín Aceña y Pedro Fraile el haber realizado una primera lectura critica de este trabajo. También agradezco las recomendaciones recibidas de dos lectores árbitros anónimos. Naturalmente, los errores son exclusivamente míos.

1 Sims (1980), p. 1. 
do enormemente el análisis, Sims opinaba que no hay una inmediata perspectiva de que los macromodelos terminen por desaparecer por una buena razón: son útiles herramientas en la predicción y el análisis de la política económica 2 . Incluso Sims, contraatacando, señalaba que seria más relevante plantear la necesidad de someter sistemáticamente las hipótesis a su contrastación empírica para ir eliminando asunciones admitidas en teoría económica que pudieran no resistir el enfrentamiento con los hechos.

Finalmente, Sims nos recuerda que, en cualquier caso, y esto es lo importante, siempre quedará la necesidad de interpretar las conclusiones econométricas, y aqui las diferentes escuelas volverán a enfrentarse inevitablemente. Así, si nos fijamos en la relación entre M (Oferta Monetaria) e Y (Renta Nominal), la prueba de la exogeneidad de Y, según Sims, podria ser interpretada de tres modos distintos:

a) Para la Escuela de las Expectativas Racionales, confirmaria que las variaciones cíclicas no son, en gran medida, una reacción a los impactos monetarios.

b) Para un monetarista clásico sería muy dificil de explicar en su marco de relaciones estructurales unidireccionales y tratará de invalidar el test.

c) Para un keynesiano clásico podría ser indicativo de la presunta ineficacia de la politica monetaria.

Por el contrario, el rechazo de la exogeneidad de $\mathrm{Y}$ debilitaría las posiciones keynesianas y las de la escuela de las expectativas racionales, reforzando la postura monetaria.

El artículo de Sims tuvo una gran repercusión, pues proponia acercar la teoria económica a la realidad, a través de la estadística, tarea en la que, a mi modo de ver, deberían estar comprometidos, de forma especial, los historiadores económicos. P. Temin nos ha explicado que «la vieja historia económica era una rama de la historia y la nueva, una rama de la economían, aunque aconseje "tender puentes" tanto con los historiadores tradicionales como con los economistas en general ${ }^{3}$.

En el presente trabajo trataremos de recoger algunos de los esfuerzos que se han hecho para aclarar la relación causa-efecto, aspecto fundamental de toda investigación, aunando las energías de los económetras y de los historiadores económicos. De este modo, en el siguiente apartado, analizaremos el problema estadístico de la causalidad, presentando una herramienta que aún

\footnotetext{
2 Sims (1980), p. 11.

3 Vid. el epilogo de Temin (1984).
} 
no ha revelado toda su utilidad: los tests de causalidad. A continuación, recordaremos la aplicación de este instrumento en historia monetaria para contrastar las predicciones de la teoria cuantitativa del dinero. Finalmente, presentaremos una aplicación al caso español, con las nuevas series macroeconómicas disponibles.

\section{EL PROBLEMA ESTADISTICO DE LA CAUSALIDAD}

En las décadas de 1950 y 1960 una serie de trabajos econométricos, entre los que destacan los de Simon, Orcutt, Strotz y Basman, pusieron de manifiesto la necesidad de estudiar el problema de la causalidad en las relaciones económicas ${ }^{4}$. La obra de Granger y Hatanaka vendría a resaltar la utilidad del análisis espectral en la descripción de las relaciones entre dos variables donde una "está causando» la otra ${ }^{5}$. Granger daría más tarde una versión más acabada del estudio ${ }^{6}$.

Granger ya advertía una primera limitación en su análisis: sólo seria aplicable a series estacionarias, pues, en otro caso, la varianza dependerá del valor de $t$ y la causalidad podría alterarse con el paso del tiempo; sin embargo, aun en este caso sería posible hablar de la relación de causalidad existente en un momento dado?.

En el mismo artículo Granger propondria su famoso «modelo de causalidad normaln: siendo $\mathrm{X}$ e $\mathrm{Y}$ dos series de tiempo estacionarias, las ecuaciones del modelo serian:

$$
\begin{aligned}
& X_{t}=\sum_{j=1}^{m} a_{j} X_{t-j}+\sum_{j=1}^{m} b_{j} Y_{t-j}+\varepsilon_{t} \\
& Y_{t}=\sum_{j=1}^{m} c_{j} X_{t-j}+\sum_{j=1}^{m} d_{j} Y_{t-j}+\tau_{t}
\end{aligned}
$$

donde $\varepsilon_{q}$ y $\tau_{t}$ son series incorrelacionadas "ruido blanco".

Aunque Granger para resolver el modelo aplicó en este artículo el análisis espectral, en la práctica, los test de causalidad han sido planteados, con más frecuencia, basándose en el análisis de regresión, a través de la aplicación de los llamados tests de causalidad de Granger/Sims. Estos tests adoptan varias formas, pero la más usual consiste en plantear una regresión que sigue el modelo Granger, siendo la hipótesis nula que $\mathrm{Y}$ no causa $\mathrm{X}$ en la primera

4 Simon (1953); Orcutt (1952); Strotz (1960); Basman (1963).

5 Granger y Hatanaka (1964).

6 Granger (1969).

? Granger (1969), p. 429. 
ecuación, y que $X$ no causa $Y$ en la segunda, es decir, que $b_{j}=0$ y $d_{j}=0, \Upsilon_{j}$, respectivamente. Alternativamente, los tests de Sims parten de una regresión de $\mathrm{Y}$ sobre los valores presentes $\mathrm{y}$ pasados de $\mathrm{X}$. La hipótesis nula que $\mathrm{X}$ causa $\mathrm{Y}$ es aceptada si la adición de los futuros valores de $\mathrm{X}$ en la regresión no mejora sensiblemente la bondad del ajuste. El mismo test es realizado en la dirección contraria para probar si Y causa X. En ambos casos se utiliza un Ftest para evaluar los resultados. También se usan habitualmente los vectores de autorregresiones múltiples, donde se comparan los resultados de todas las regresiones posibles entre las variables elegidas, introduciendo retardos en las variables exógenas.

\section{LA APLICACION DE LOS TESTS DE CAUSALIDAD EN HISTORIA MONETARIA}

Desde que la Nueva Historia Económica apareció, a finales de la década de 1950, se ha extendido entre los historiadores el uso de la econometría, como instrumento de contrastación de las hipótesis que plantea la teoría. La complejidad que presentan estas técnicas para investigadores no familiarizados con ellas ha quedado disminuido, en gran medida, por la disponibilidad de una serie de programas de ordenador introducidos en los años sesenta. Van der Wee ha llegado a afirmar que con la simulación por ordenador ha quedado resuelto el viejo problema de la imposibilidad de los experimentos sociales, aunque advierte que «siendo indefinido el análisis exhaustivo del factor residual, todo modelo explicativo permanece en el estado de causa provisional" 8 .

Hemos tenido ocasión, en el capitulo anterior, de hacer una breve presentación de la aparición y desarrollo de los tests de causalidad en el universo de la estadistica teórica. Era lógico esperar que este tipo de contrastes no pasara desapercibido para los historiadores, al ser el desvelamiento de la relación causa-efecto parte fundamental en su campo de estudio. De este modo, desde finales de la década de 1970, aparecieron, principalmente en los Estados Unidos, una serie de trabajos, centrados en el campo de la historia monetaria, y que van a intentar dar respuesta, por la vía empírica, a la gran controversia monetarismo-keynesianismo del momento.

Se trataba, pues, de determinar si las fuerzas monetarias eran la causa de los movimientos de la renta nominal, los precios y el output real como apuntan Friedman-Schwartz (1963), o si, por el contrario, los movimientos de la cantidad de dinero eran determinados desde la economia real como

8 Vid. Temin (1974). 
postulaban sus críticos - Tobin, Kaldor- El cuadro siguiente presenta, de forma ordenada, las conclusiones de los principales estudios que sobre esta cuestión se hicieron, aplicando los tests de causalidad:

\section{CUADRO 1}

Cuadro-resumen de la aplicación de los tests de causalidad en bistoria monetaria

\begin{tabular}{|c|c|c|c|c|}
\hline Obra & Variables & Ambito & Metodologia & Conclusiones \\
\hline $\begin{array}{l}\text { Mills-Wood } \\
\text { (1978) }\end{array}$ & $\mathrm{M}, \mathrm{Y}, \mathbf{r}, \mathrm{P}$ & $\begin{array}{l}\text { U.K., } \\
\text { 1870-1914 }\end{array}$ & Tests de Sims & $\begin{array}{l}\text { Y,r,P exógenas } \\
\mathrm{Y} \Rightarrow \mathrm{M} \\
\text { Importante papel de los } \\
\text { flujos internacionales de } \\
\text { oro }\end{array}$ \\
\hline $\begin{array}{l}\text { Brillembourg } \\
\text { Khan (1979 }\end{array}$ & $\mathrm{M}, \mathrm{Y}, \mathrm{P}$ & $\begin{array}{l}\text { U.S.A., } \\
1870-1975\end{array}$ & Tests de Sims & $\begin{array}{l}\text { No hay independencia } \\
M \Rightarrow Y, P ; \text { adelanto }=2 \\
\text { años } \\
\text { Relación no estable, va- } \\
\text { ría historicamente }\end{array}$ \\
\hline $\begin{array}{l}\text { Huffman- } \\
\text { Lothian (1980) }\end{array}$ & $\mathrm{H}, \mathrm{Y}$ & $\begin{array}{l}\text { U.K., } \\
1837-1968\end{array}$ & Tests de Granger & $\begin{array}{l}\text { En } 1837-1870, H \Rightarrow Y \\
\text { Interrelación en el resto } \\
\text { Los flujos de oro no de- } \\
\text { terminaron } H \text { en } 1837 \text { - } \\
1870\end{array}$ \\
\hline $\begin{array}{l}\text { Eichengreen } \\
\text { (1983) }\end{array}$ & $\mathrm{H}, \mathrm{Y}, \mathrm{P}, \mathrm{X}, \mathrm{Iv}$ & $\begin{array}{l}\text { U.K., } \\
\text { 1833-1913 }\end{array}$ & $\begin{array}{l}\text { Vector de autorre- } \\
\text { gresiones }\end{array}$ & $\begin{array}{l}\text { Las «fuerzas reales» más } \\
\text { importantes antes de } \\
1880 \\
\text { Las «fuerzas moneta- } \\
\text { rias" más importantes } \\
\text { después }\end{array}$ \\
\hline Dwyer (1985) & $\mathrm{M}, \mathrm{P}, \mathrm{Y}, \mathbf{r}$ & $\begin{array}{l}\text { U.K., } \\
\text { 1870-1913 }\end{array}$ & $\begin{array}{l}\text { Vector de autorre } \\
\text { gresiones }\end{array}$ & $\begin{array}{l}\text { Md } \Rightarrow M \\
\text { U.K. es un pais upe- } \\
\text { queñow en el periodo, } \\
\text { influido por la política } \\
\text { de USA }\end{array}$ \\
\hline
\end{tabular}

$M=$ Oferta Monetaria

$\mathrm{Y}=$ Renta

$r=$ Tipo de interés

$\mathrm{P}=$ Nivel general de precios

$\mathrm{H}=$ Base Monetaria

$\mathrm{X}=$ Exportaciones

Iv $=$ Construcción de viviendas

Md = Demanda de dinero

FUENTE: Elaboración a partir de los artículos citados en la bibliografia. 
Conviene recordar cómo, a finales de la década de 1970, era comúnmente aceptada la proposición de Friedman-Schwartz acerca del papel del dinero como factor explicativo, tanto en los movimientos cíclicos como en el largo plazo. Sin embargo, la inmensa mayoría de los estudios no habían ido más allá de establecer la correlación entre las variables reales y las monetarias, obviando el principio de que la correlación no implica causalidad, y aceptando una causalidad unidireccional, en el sentido apuntado por FriedmanSchwartz: $M \Rightarrow P$.

Entre los pocos estudios precisos disponibles, el trabajo de Feige-Pearce se enfrentaba con el de Sims, y ambos trabajaban sólo con datos de la economía norteamericana posteriores a 1945. Sims encontraba una causalidad unidireccional del dinero a la renta, mientras que para Feige-Pearce el nivel de precios era, en gran medida, independiente de las variaciones monetarias y la relación entre la cantidad de dinero y la renta nominal mucho más débil de lo indicado por Sims.

En 1978, en la revista del Banco de la Reserva Federal de San Luis, aparecerá el estudio de Mills-Wood sobre la economía británica del período 1870-1914 que encuentra un efecto significativo de la renta sobre la oferta monetaria, pero no al revés. Los resultados venían a confirmar los obtenidos anteriormente por McCloskey-Zecher.

Ante los resultados de estas nuevas investigaciones, Brillembourg-Khan afirmarán que ula naturaleza de la relación causal entre el dinero, los precios y la renta ha sido recientemente investigada y sus conclusiones han resultado conflictivas" ". Sin embargo, ampliando el período de investigación de Sims y Feige-Pearce, Brillembourg-Khan estudian los datos norteamericanos del período 1870-1975. El dinero aparecerá determinando la renta nominal y los precios, con un adelanto de dos años aproximadamente. Sin embargo, al estudiar la estabilidad de esta relación causal, considerando tres subperíodos (1870-1913, 1914-1945 y 1946-1975), los tests de Sims no van a poder aclarar la incidencia de los factores monetarios en la Gran Depresión y para la posguerra de 1945 no habrá constatación empírica de la relación entre el dinero y la renta real, ni entre el dinero y los precios.

Al año siguiente, Huffman-Lothian ampliarán el controvertido estudio de Mills-Wood analizando el período 1837-1968, para la economía británica. Por otra parte, deciden reducir la definición de dinero a la base monetaria, pues, en su opinión, no había datos fiables de depósitos para todo el periodo. Ello, sin embargo, planteará importantes problemas a la hora de comparar los

- Brillembourg-Khan (1979), p. 358. 
resultados con los obtenidos por Mills-Wood. También diferirá el estudio de Huffman-Lothian respecto de su predecesor en la metodología de los tests de causalidad. Huffman-Lothian se inclinan por los tests de Granger frente a los de Sims, al considerar más econométrico el procedimiento de no utilizar valores futuros en las variables tomadas como exógenas ${ }^{10}$.

Las conclusiones serán igualmente controvertidas: «Sobre el periodo de mayor interés, 1837-1870, la dirección de influencia no tiene ambigüedades: de la base monetaria a la renta nominal. En el periodo 1870-1914 la relación es muy débil en ambas direcciones. Finalmente, en el período $1914-1960$ y el total del período 1837-1968 la influencia corre en ambas direcciones» ${ }^{11}$.

¿Por qué la influencia de la base monetaria sobre la renta nominal es tan clara en 1837-1870 a diferencia de lo que ocurrirá después? Huffman-Lothian piensan que «el Reino Unido en este tiempo desempeñó un papel análogo al de los Estados Unidos durante la era de Bretton Woods» ${ }^{12}$, y su mayor tamaño relativo le permitió imponer un patrón oro británico que mantuviese la discrecionalidad en la determinación de la base monetaria. Esta sería una explicación a su exogeneidad.

En cualquier caso, la heterodoxia del caso inglés volvía a ponerse de manifiesto y los estudios siguientes se centrarian en tratar de darle una explicación. Era de esperar que en una época de revitalización de la teoria cuantitativa del dinero estos resultados no pudieran admitirse fácilmente.

Eichengreen introducirá más variables para tratar de completar el análisis. Junto a las anteriores, aparecen las exportaciones y la construcción residencial. Su metodología se basa en el uso de los vectores de autorregresión. Los resultados serán sorprendentes: las fuerzas de la economía real se revelan dominantes antes de 1880 , mientras que las fuerzas monetarias serán determinantes desde entonces.

Finalmente, Dwyer, que curiosamente parece ignorar el estudio de Eichengreen, "extiende el análisis separando renta real y nivel de precios e incluyendo los tipos de interés» ${ }^{13}$, situándose en el periodo 1870-1914. Utiliza, asimismo, la metodología de los vectores de autorregresiones:

$$
\mathrm{X}=\alpha_{j} \mathrm{X}_{-j}+
$$

10 Huffman-Lothian (1980), pp. 156 y 162.

1 Huffman-Lothian (1980), p. 164.

12 Huffman-Lothian (1980), p. 165.

13 Dwyer (1985), p. 416. 
donde $\alpha$ es una matriz de coeficientes $(4 \times 4)$ y $\varepsilon$ es un vector de perturbaciones $(4 \times 1)$. $X$ es un vector $(4 \times 1)$ de las cuatro variables en el periodo $t(M$, $\mathrm{P}, \mathrm{Y}, \mathrm{r})$. Tres retardos le parecen adecuados para caracterizar los datos.

Dwyer encuentra que «la hipótesis de que el Reino Unido es un pais pequeño, es decir, que el nivel de precios y los tipos de interés son determinados independientemente de los valores pasados de la renta real y de la oferta monetaria en el Reino Unido, no puede ser rechazada a los niveles de significación habituales» ${ }^{14}$. Para Dwyer esto sería consistente con lo hallado por Brillembourg-Khan para los Estados Unidos, que serian, de este modo, un país "grande» en el periodo estudiado.

Hay que señalar que Huffman-Lothian ya habian señalado las diferencias entre los modelos económicos del Reino Unido y los Estados Unidos, antes y después de la guerra civil americana, concluyendo que la influencia de Estados Unidos sobre el Reino Unido es relativamente más importante a finales del siglo XIX y principios del siglo XX que en la primera mitad del siglo XIX.

Finalmente, Bordo, resumiendo el estado de la cuestión, expresa que la información sugiere, para el Reino Unido, causación inversa de la renta real al dinero, consistente con el enfoque monetario de la balanza de pagos (M. A. B. P.). Este resultado puede ser debido al carácter de economia abierta del Reino Unido ${ }^{15}$. Como es sabido, de acuerdo con la M. A. B. P., los precios y los tipos de interés están fuertemente ligados a través del arbitraje en los mercados de bienes y capitales, siendo los flujos de oro el mecanismo equilibrador que compensa los excesos de demanda u oferta del dinero.

De este modo, como también señalan McCloskey-Zecher, si la economía mundial está unificada por el arbitraje y el nivel mundial de precios es determinado por la oferta mundial de dinero, entonces el banco central de un país «pequeño" adherido al patrón oro podría controlar sólo la composición de la base monetaria, dividida en reservas internacionales y crédito interno, pero no su importe total. La oferta de dinero seria una variable endógena, influida poderosamente por los flujos internacionales de oro y la política monetaria vería muy limitada su eficacia.

Sin embargo, recientemente Friedman-Schwartz han replicado que el régimen del tipo de cambio no condiciona la influencia causal del dinero sobre la renta; afecta, sin embargo, a las fuerzas determinantes de la cantidad de dinero y sobre si la relación se trata de una influencia unidireccional amplia

14 Dwyer (1986), p. 425

15 Bordo (1986), p. 350. 
del dinero a la renta o de una interacción y determinación simultánea ${ }^{16}$. Quizá esta última afirmación pueda ser una prueba de cómo el análisis cliométrico puede ayudar a poner en tela de juicio afirmaciones simplistas del tipo $M \Rightarrow P$, que extendió la moderna teoría cuantitativa del dinero. Las relaciones entre el sector real y el sector monetario de la economía parecen ser mucho más complejas de lo que pretende la metodologia instrumentalista.

\section{TESTS DE CAUSALIDAD, DINERO Y RENTA EN ESPANA}

Como ha quedado señalado en la sección anterior, los tests de causalidad son una metodología bastante moderna, que ha sido aplicada en historia monetaria para dar luz en la polémica sobre la relación entre las variables monetarias y las variables reales de la economia.

Su uso se ha extendido en el mundo anglosajón, más inclinado hacia el análisis cliométrico, y los trabajos más relevantes se han centrado en los casos de Estados Unidos y el Reino Unido, paises para los que se dispone, además, de estadísticas monetarias de cierta fiabilidad desde hace tiempo. En España, la reciente publicación de Carreras, que recoge un esfuerzo muy meritorio de revisión y ordenación de nuestros principales datos históricos va a permitir, con seguridad, la profusión de los estudios cuantitativos.

En este trabajo se realiza una aproximación al análisis de la causalidad entre la renta nominal (Y) y la cantidad de dinero (M). El trabajo incluye datos desde 1904 a 1974, lo que nos permitirá comparar la relación entre M e $Y$ en diferentes periodos. Se han realizado sendos tests utilizando dos fuentes para la serie de renta nominal: el cuadro 2 utiliza los conocidos datos de Alcaide y el cuadro 3 se basa en la serie de Carreras sobre el gasto nacional.

El análisis de causalidad empleado sigue estrechamente el trabajo de Huffman-Lothian, que está basado en el modelo de causalidad normal de Granger, expuesto anteriormente, y que podemos resumir en la ecuación:

$$
X_{t}=a+\sum_{k=1}^{m} b_{k} X_{t-k}+\sum_{k=1}^{m} c_{k} Z_{t-k}+\varepsilon_{t}
$$

Renta nominal y cantidad de dinero alternan como variables dependiente e independiente. En un primer conjunto de regresiones se ha realizado la

16 Friedman-Schwartz (1982), p. 325. 
transformación logarítmica de ambas variables, incluyendo una variable de tendencia y la constante, haciendo $m=3$. En el segundo conjunto de regresiones, hemos introducido las primeras diferencias de los logaritmos de las variables, añadiendo una constante, $y$ haciendo $m=2$. En cada ocasión hemos contrastado la hipótesis nula de que $c_{k}=0, \Upsilon_{k}$, es decir, que $Z$ no causa $X$, en el sentido de Granger.

Hemos dividido el período completo en tres subperíodos convencionales: 1904-1918, 1919-1935 y 1944-1974, a fin de obtener información sobre la estabilidad de la relación entre variables monetarias y reales que vayamos encontrando.

A la vista de los resultados del cuadro 2 , no podemos rechazar la hipótesis nula en ninguno de los dos sentidos, a un nivel de significación del 5 por 100 , por lo que la influencia aparece ambigua. Sin embargo, el test obtiene valores más altos en la relación $M \Rightarrow Y$ que en la inversa.

\section{CUADRO 2}

Relación entre la cantidad de dinero y la renta nominal 1904-1974 y subperiodos. Renta tomada de Alcaide

\begin{tabular}{ccccc}
\hline Periodo & $\begin{array}{c}\text { Variable } \\
\text { Dependiente }\end{array}$ & F-test & $\begin{array}{c}\text { Variable } \\
\text { Dependiente }\end{array}$ & F-test \\
\hline $1904-1974$ & L.Y & $1,87(2,78)$ & LM & 0,15 \\
$1904-1918$ & & $3,79(4,35)$ & & 1,04 \\
$1919-1935$ & & $0,93(3,86)$ & & 0,82 \\
$1944-1974$ & & $0,99(3,01)$ & & 0,18 \\
$1904-1974$ & GLY & $1,14(3,16)$ & \\
$1904-1918$ & & $2,35(4,10)$ & & 0,29 \\
$1919-1935$ & & $2,11(3,89)$ & & 1,87 \\
$1944-1974$ & & $1,02(3,37)$ & & 0,08 \\
\end{tabular}

LY $=$ Logaritmo natural de la renta nominal

LM = Logaritmo natural de M3

GLY = Primeras diferencias logaritmicas de la renta nominal

GLM = Primeras diferencias logaritmicas de M3

Los valores críticos en cada test para alfa $=0,05$ van indicados entre paréntesis.

Fuente DE DATOS: Carreras (coord) (1989).

Por el contrario, los resultados del cuadro 3 permiten rechazar la hipótesis nula de no causalidad en 1944-1958 con claridad, afirmando que $\mathrm{Y} \Rightarrow \mathrm{M}$ durante este periodo. Asimismo, los resultados del F-test son siempre más 
altos en la relación $Y \Rightarrow M$, aunque no se llegue a superar el valor crítico, excepto en el periodo señalado y para GLM en 1904-1918.

\section{CUADRO 3}

Relación entre la cantidad de dinero y la renta nominal 1904-1958 y subperiodos. Renta tomada de Carreras

\begin{tabular}{lcccc}
\hline Periodo & $\begin{array}{c}\text { Variable } \\
\text { Dependiente }\end{array}$ & F-test & $\begin{array}{c}\text { Variable } \\
\text { Dependiente }\end{array}$ & F-test \\
\hline $1904-1958$ & LY & $0,08(2,85)$ & LM & 1,57 \\
$1904-1918$ & & $2,96(4,35)$ & & 3,02 \\
$1919-1935$ & & $0,68(3,86)$ & & 1,97 \\
$1944-1958$ & & $0,88(4,35)$ & & 5,26 \\
$1904-1958$ & GLY & $0,79(3,21)$ & GLM & 1,74 \\
$1904-1918$ & & $0,27(4,10)$ & & 6,12 \\
$1919-1935$ & & $0,43(3,89)$ & & 1,56 \\
$1944-1958$ & & $0,45(4,10)$ & & 4,44 \\
\hline
\end{tabular}

LY $=$ Logaritmo natural de la renta nominal

$L M=$ Logaritmo natural de $M 3$

$G L Y=$ Primeras diferencias logaritmicas de la renta nominal

GLM = Primeras diferencias logaritmicas de M3

Los valores criticos en cada test para alfa $=0,05$ van indicados entre paréntesis.

FUENTE DE DATOS: Carreras (coord) (1989).

Por otro lado, el cuadro 4 nos indica que la correlación entre renta nominal y M3 es considerablemente más alta con los datos de renta de Alcaide que con los datos de Carreras.

\section{CONSIDERACIONES FINALES}

Bordo ha señalado, acertadamente, que el uso de los tests de causalidad es una aportación bienvenida a la "caja de herramientas" de los historiadores económicos, aunque, «como en todas las nuevas tecnologías, sus limitaciones deben ser tenidas en cuenta» ${ }^{17}$. Entre éstas, cabe destacar que al excluir otras variables explicativas en las ecuaciones se omite el problema de la correlación

17 Bordo (1986), p. 350. 
CUADRO 4

Coeficientes de correlación entre la renta nominal y M3 1904-1974 y subperiodos

\begin{tabular}{ccc}
\hline Periodo & YALC/M3 & YCAR/M3 \\
\hline $1904-1974$ & 0,93 & 0,70 \\
$1904-1918$ & 0,77 & 0,53 \\
$1919-1935$ & 0,95 & 0,66 \\
$1944-1974$ & 0,94 & 0,93 \\
\hline
\end{tabular}

YALC = Renta nominal tomada de Alcaide (1976)

YCAR = Renta nominal tomada de Carreras (1989)

M3 = Oferta Monetaria

FUENTE DE DATOS: Carreras (coord) (1989)

parcial, con lo que los resultados presentan sesgos estadísticos importantes.

También es muy atinada la crítica de Zellner y Leamer, que insiste en distinguir "antecedente temporal» y "causa», sobre todo si el análisis se realiza con modelos sin interpretación teórica. A pesar de todo, se sigue extendiendo su uso, y los recientes trabajos de Stock-Watson y Pikkarainen-Viren son buena prueba de ello.

Para el caso español, los resultados que hemos obtenido han resultado ser contradictorios y no nos permiten concluir una influencia unidireccional en la relación entre variables monetarias y reales. Que la información empírica sea tan confusa no debe sorprendernos demasiado, si tenemos en cuenta las conclusiones obtenidas en otros paises. Además, los resultados alcanzados, aplicando tests de causalidad a la economia española entre 1874 y 1935 por Anes, y con datos contemporáneos por Hoyo-Terceiro también apuntan la debilidad de las relaciones existentes entre las variables macroeconómicas. Es destacable que Anes utilizó como indicador de la evolución del sector real de la economía, el transporte de mercancías por ferrocarril, comparándolo con la evolución de la oferta monetaria.

Por otro lado, creo importante señalar que la divergencia entre los Cuadros 2 y 3 puede atribuirse a la heterogeneidad de los datos de renta usados en cada caso. Como ha señalado Carreras, su serie de gasto nacional sufre de una excesiva irregularidad, fruto del carácter agregativo (como la del CEN) y no estimativo (como la de Alcaide) del cálculo» 18. La mayor

18 Carreras (1989), p. 541. 
variabilidad de los datos de Carreras debe tener consecuencias importantes en el análisis aquí realizado. Esto viene a confirmar la necesidad de seguir mejorando nuestras series de renta, pues el uso de las actuales tendrá que terminar por ser forzosamente alternativo en los estudios cliométricos.

\section{BIBLIOGRAFIA}

AlCAIDE, J. (1976): “Una revisión urgente de la serie de renta nacional española en el siglo XX", en Datos básicos para la bistoria financiera de Espaĩa, 1850-1975, vol. I, Instituto de Estudios Fiscales.

ANES, R. (1978): "Comparación de las fluctuaciones monetaria y real de la economia española (1874-1935)", en OTAZU, A. (ed.): Dinero y Crédito (siglos XVI al XIX), Madrid.

BASMAN, R. L. (1963): "The Causal Interpretation of Non Triangular Systems of Economic Relations», Ecomometrica, vol. 31.

BORDO, M. D. (1986): «Explorations in Monetary History: A Survey of the Literaturem, Explorations in Economic History, vol. 23.

BRILlembouRG, A., y KAHN, M. S. (1979): "The Relationship Between Money, Income and Prices: Has Money Mattered Historically?w, Journal of Money, Credit and Banking, vol. 9.

CARreras, A. (coord.) (1989). Estadisticas bistoricas de Espaita. Siglos XIX-XX, Fundación Banco Exterior.

EICHENGReEN, B. (1983): "The Cause of British Business Cycles, 1833-1913", Tbe Journal of European Economic History, vol. 12.

Feige, E. L., y PEARCE, D. K. (1974): Tbe Causality Relationship Between Money and Income: $A$ Time Series Approacb, Chicago.

Friedman, M., y SCHWARTZ, A. J. (1963): A Monetary History of the United States, 1867-1960, Princeton.

- - (1982). Monetary Trends in the United States and the United Kingdom, Chicago. Granger, C. W. J., y Hatanaka, M. (1964). Spectral Analysis of Ecomomic Time Series, Nueva York.

(1969): «Investigating Causal Relationships by Econometric Models and Cross Spectral Methodsm, Econometrica, vol. 37.

Harvey, A. C. (1981): The Econometric Analysis of Time Series, Nueva York.

HOYO, J. DEL, y TERCEIRO, J. (1979): «Causalidad en series temporales. Alguna evidencia empirica para la economía españolan, Cuadernos de Economía, vol. 6, núm. 17.

Huffman, W. E., y Lothian, J. R. (1980): «Money in the United Kingdom, 18331880", Journal of Money, Credit and Banking, vol. 12.

KALDOR, N. (1970): "The New Monetarism», Lloyd's Bank Review, núm. 1.

LEAMER, E. (1985): «Vector Autoregression for Causal Inference», Carnogie Rocbester Conference. Series on Pablic Poligy, vol. 22.

MC Closkey, D. N., y ZECHER, R. J. (1976): «How the Gold Standard Worked, 1880-1913?», Frenkel, J. A., y JOHNSON, H. G. (ed.): Tbe Monetary Approacb to the Balance of Payments, Toronto. 
Mills, T. C., y WOOD, G. E. (1978): «Money-Income Relationships and the Exchange Rate Regimes, Federal Reserve Bank of St. Lowis Review, vol. 60. y - (1982): «Econometric Evaluation of Alternative Money Stock Series, 1880-1913", Jownal of Money, Credit and Banking, vol. 14.

OrcutT, G. H. (1952): «Actions, Consequences and Causal Relations», Review of Economics and Statistics, vol. 34.

Pikkarainen, P., y Viren, M. (1989): «Granger Causality Between Money, Output, Prices and Interest Rates. Some Cross-Country Evidence from the Period 18751984", Weitwirtscbaftlicbes Arcbiv, vol. 125.

SimON, H. A. (1953): "Causal Ordering and Identificability", HoOD, W. C., y Koopmans, T. C. (ed.): Studies in Econometric Metbod, Cowles Commission Monograph, vol. 14.

Sims, C. A. (1972): «Money, Income and Causalitym, American Economic Review, vol. 62.

(1980): «Macroeconomics and Reality» en Econometrica, vol. 48.

STOCK, J. H., y WATSON, M. W. (1989): «Interpreting the Evidence on MoneyIncome Causalityn, Jowrnal of Econometrics, núm. 40.

STROTZ, R. H., y WOLD, H. (1960): «Recursive versus Non-Recursive Systems: An Attempt of Synthesis», Econometrica, vol. 28.

TEMIN, P. (1984): La nueva bistoria económica, Madrid.

TOBIN, J. (1970): «Money and Income: Post Hoc Ergo Propter Hoc?», Qwarterly Jowrnal of Economics, vol. 84 (con comentario crítico de M. Friedman).

ZELLNER, A. (1989): «Causality and Econometricsm, Carnogie Rocbester Conference. Series on Public Poligy, vol. 10.

V. V. A. A. (1974): El método bistórico, Univ. de Navarra. 